\title{
Stem Mutants in the N-terminal Domain of the Phage P22 Tailspike Protein
}

\author{
Chris Palmer ${ }^{1}$, Jeremie Williams ${ }^{1}$, Dexter Dean ${ }^{2,3}$, Sam Johnson ${ }^{1}$, Hongzhuan $\mathrm{Wu}^{1}$, Boakai K. Robertson ${ }^{1}$, Doba \\ Jackson $^{2}$, Robert Villafane ${ }^{1, *}$ \\ ${ }^{1}$ Program in Microbiology, Alabama State University, Montgomery, AL, USA \\ ${ }^{2}$ Department of Chemistry and Biochemistry, Huntingdon College, Montgomery, AL, USA \\ ${ }^{3}$ Department of Chemistry and Biochemistry, University of Southern Mississippi, Hattiesburg, MS, USA \\ *Corresponding author: rvillafane@alasu.edu.
}

Received August 12, 2013; Revised December 10, 2013; Accepted December 26, 2013

\begin{abstract}
The P22 tailspike protein is an intensely studied protein whose structure and sequence has been described. However, a study, describing important protein interactions related to its function at the N-terminal domain, has been lacking. The P22 tailspike protein (TSP) consists of three identical polypeptide chains of 666aa. The first 108 of the 666aa in the P22 TSP form a trimeric N-terminal domain (NTD). Each of the three chains of the trimeric NTD contributes to the formation of a dome-like structure. Our studies suggest that a short stretch of amino acids located within the first fifteen amino acids of the P22 TSP NTD is critical for the stability of the dome structure formed by the first 108aa of the P22 TSP NTD. The first 23aa are located within this dome-like structure and have been dubbed the "stem" of the NTD. Although amino acid residues in the first 15aa (lower stem) are critical, deletion analysis and in vitro assembly studies implicate the rest of the stem in additional stabilizing interactions. Our studies implicate a common protein-protein interaction motif made up of interchain hydrophobic contacts between adjacent chains
\end{abstract}

Keywords: protein-protein interactions, Salmonella, tailspike protein, phage assembly

Cite This Article: Chris Palmer, Jeremie Williams, Dexter Dean, Sam Johnson, Hongzhuan Wu, Boakai K. Robertson, Doba Jackson, and Robert Villafane, "Stem Mutants in the N-terminal domain of the Phage P22 Tailspike Protein.” American Journal of Microbiological Research 2, no. 1 (2014): 1-7. doi: 10.12691/ajmr-2-1-1.

\section{Introduction}

\subsection{The Structural Domains Of The $P 22$ Tailspike Protein}

The P22 phage is a lambdoid bacterial virus which infects a very limited number of Salmonella species including Salmonella typhimurium [1,2]. The P22 phage has an ordered assembly pathway for its capsid and tail assembly processes $[3,4,5]$. The last protein that is assembled intracellularly onto the virus structure is the tail protein (tailspike protein, TSP) of the phage [5]. The structure of the P22 trimeric TSP has been described in detail in $\mathrm{X}$-ray crystallographic and cryoelectron microscopy studies, Figure 1 (Pymol representation of $\mathrm{x}$ ray coordinates) [6-11]. The P22 TSP consists of three structural elements: 1) The P22 TSP N-terminal domain (P22 TSP NTD), containing the first 108 amino acids (aa1-aa108) from each of three TSPs, exists as a domelike structure, Figure 2 [9]. The P22 TSP NTD is necessary for P22 phage particle assembly [12]. 2) The second domain, the beta helix domain, contains amino acids, aa140 to aa543. TSP binding to lipopolysaccharide (LPS) and TSP endorhamnosidase enzymatic activity on LPS, are located in the beta helix domain Figure 1. 3) The third domain, the trimerization domain, contains aa570aa666 [10]. It is in this third domain that the three separate identical polypeptide chains become interdigitated.

\subsection{Structure and Function in the P22 TSP NTD}

The major function of the P22 TSP NTD is the binding of NTD to the tailless phage (phage head) to complete the assembly of the phage particle. The P22 TSP NTD binds to a crevice formed by viral structural proteins GP4 and GP10 [7,9]. This last assembly step provides the basis for an easy and qualitative Petri plate assay, the Berget Assay [13], used to determine if a particular TSP clone contains a functional tail gene. The P22 NTD has been shown to be homologous to the NTDs of other Salmonella phage tails [14].

The topological area where the three chains meet at the top of the dome is herein called the "stem region". Each polypeptide chain contributes a 23aa sequence that goes from the top of the dome through the dome interior and these small segments of the chains are called "stems". Phenylalanine 23 (PHE23) is the stem amino acid located at the top of the dome while Methionine (MET1) is located at the dome bottom. The protein structure in the interior of the dome for each chain is called the "stem wall”. The crystal structure of the P22 TSP NTD reveals that part of each stem is located near their cognate chains, upper stem (aa13-aa23), while the other part of it, the 
lower stem (aa1-aa12) is located very close to the adjacent chain, Figure 2 [9,11]. The first five amino acids are likely to be mobile since they are not visible in these $\mathrm{x}$-ray structures [9].

This part of the P22 TSP takes on a dome-like structure where the dome is made up of each of the three chains. There are no covalent bonds between these chains and its function depends on the dome integrity. By using the Pymol visualization program on this dome structure, it was observed that there was a peptide between adjacent chains that might serve to connect each of the chains [11]. In Figure 2, the three identical chains in the NTD dome are here distinguished from one another by their colors, red (chain D), green (chain E) and yellow (chain F) in this figure. Each chain contains two sets of beta sheets which form the "walls" for this dome structure [9]. Each chain also contains a loop-like structure which we term the stem which starts at the dome top and ends up in the adjacent chain. This is most readily seen in the chain $\mathrm{D}$ where the stem extends to the right and ends within the dome interior next to the adjacent chain $\mathrm{F}$. A valine residue (blue sticks), can be seen which is part of the $\mathrm{D}$ chain but located in chain F. About half of stem D (aa16-aa23 called the upper stem) is next to the wall D but about half (aa115 called the lower stem) is located very near wall $\mathrm{F}$. PHE23 is located at the dome top next to its cognate chain. Previous studies have suggested that the three PHE23s at the dome top from each of the chains results in the stabilization of the NTD [9]. However, the lower stems, from each of these chains, seem a more attractive linking segment.

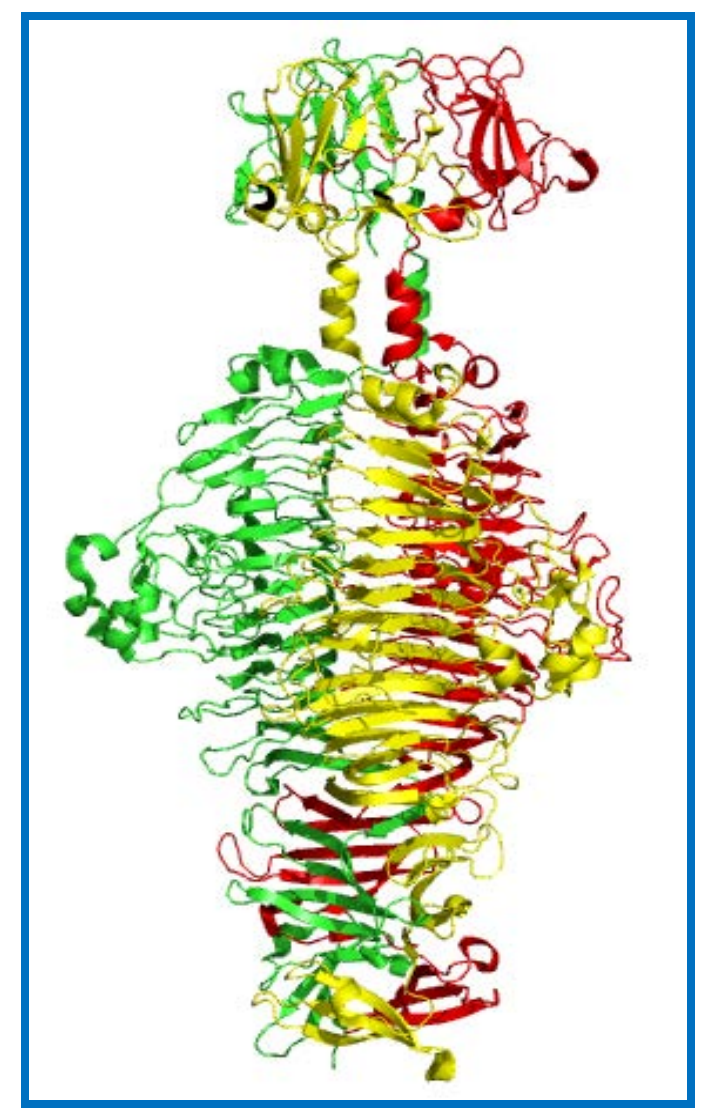

Figure 1. Pymol rendering of the trimeric structure of the P22 TSP from $\mathrm{X}$-ray structural coordinates in 2xc1 $[9,10]$. Coordinates were obtained from the RCSB. Chains D, E and F are color-coded as red, green and yellow, respectively. This structure is a model from two solved structures: the NTD and the CTD which are here modeled together

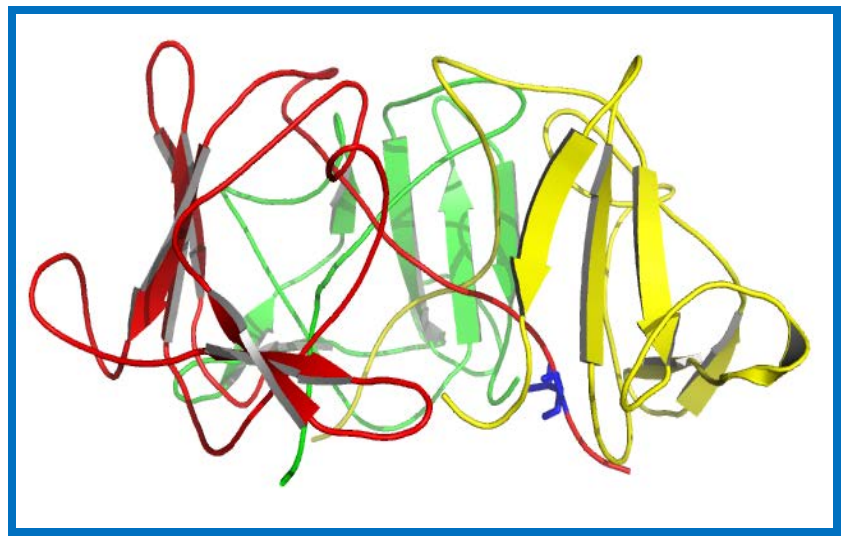

Figure 2. Pymol rendering of the trimeric N-terminal domain of the P22 TSP from X-ray structural coordinates $[9,10]$. Chains D, E and F are color-coded as red, green and yellow, respectively. Also evident is and located in the $\mathrm{E}$ chain at the bottom right is a blue stick representing the valine 9 from the $\mathrm{D}$ chain

Hydrophobic amino acids have long been known to be the major driving source for binding interactions involved in protein interactions, stability and folding [25-32]. Such an interaction would require that the stem and the putative interacting partner, the adjacent wall, both contain hydrophobic amino acids. Of the first 10 amino acids of the lower stem, four are hydrophobic: an alanine amino acid residue at position six (ALA6) and three valines at positions 8, 9 and 10 (VAL8, VAL9, and VAL10). It is hypothesized that the three valine hydrophobic residues, form a hydrophobic patch, attaching the $\mathrm{D}$ chain to the $\mathrm{F}$ chain and every stem with its adjacent protein chain and that this patch is the main source of stability for the trimerization of the P22 TSP NTD. The ALA6 is less likely to be involved in this stabilization especially since it is located near a mobile set of amino acids, aa1-aa5.

This report describes the initial studies in the structure and function analyses of the P22 TSP NTD with particular interests in the protein-protein interactions involving this domain. The study reported here suggests that one such protein-protein interaction involving aa1-aa23 is critical for the stability and structural integrity of the P22 TSP NTD.

\section{Materials and Methods}

\subsection{Bacterial Strains and Plasmids}

The Escherichia coli cells used in this study were from Novagen. These strains were: NovaBlue Singles which is $\mathrm{K}-12$ recA1 endA, lacIq and BL21(DE3) which is E. coliB strain F- ompT (lon) hsdS (rB-mB-) with prophage carrying the gene for T7 RNA polymerase also from Novagen. BL21 (DE3/pET11a-TSP) was courtesy of Dr Peter Prevelige (University of Alabama at Birmingham, Microbiology Department). The plasmid pET11a-TSP was constructed by Drs Anne Robinson and Jon King and described [15]. This plasmid, pET11a-TSP, is our source plasmid containing the cloned wild type version of the P22 tail gene, from which all mutant derivatives in this study were derived. Other bacterial strains were generous gifts of Dr Jon King (MIT, Biology Department, Cambridge, MA). Phage heads (phage particles missing only the tail or tailspike protein) were prepared from DB7136 (9-amN110/ 13-amH101/ c2ts30) and was a gift from Dr Jon King. 


\subsection{General Microbiological Protocols}

General bacterial and bacteriophage procedures such as monitoring bacterial growth, preparation of plasmids and phages, transformation of plasmids, titering phage (determination of phage concentration), mutagenesis of phage or plasmids etc have been described [16,17].

\subsection{In Vitro Tailing Assay}

Addition of phage heads, either purified or in extract forms, to tails, either purified or in extract forms, at $4{ }^{\circ} \mathrm{C}$ for 30min will cause the tails to bind to the tailless phage particles to form intact complete infectious phage particles in vitro $[18,19]$. This is called in vitro tailing or in vitro assembly.

\subsection{In Vivo Tailing Assay (Berget Assay)}

If a colony of Escherichia coli (with a resident plasmid vector containing a cloned P22 tailspike gene) is placed on the surface of the solidified top agar of an LB petri plate (containing phage heads plus Salmonella typhimurium [ST] cells), then after overnight incubation at $30^{\circ} \mathrm{C}$ each $E$. coli colony would be surrounded by an area or zone of lysis. This area of lysis is presumably due to the leakage of P22 TSP from lysed cells within the growing colony during overnight growth of the colony. The P22 TSP diffuses away from the lysed cells of the colony and encounters phage heads and ST outside of the colony. The last step of assembly occurs outside of an E. coli colony and an infectious phage is generated which immediately finds $S T$ and lyses them outside of the colony. The result is the production of an area or zone of lysis around the E. coli colony that contains a clone with an actively expressed tail gene. This assay allows for the determination of the functional state of a cloned tailspike gene or variant in $E$. coli [13]. It was shown that the area of lysis around an $E$. coli cell, containing a tail-clone, was indicative of its function. The lack of zones or halos of lysis around an $E$. coli colony, (carrying a cloned TSP gene), indicates a lack of TSP function. This indicated that although the in vivo plate tailing assay (dubbed the Berget Assay in this report [13] is a plate assay that is quite sensitive.

This in vivo tailing assay has been modified by this laboratory to allow the discrimination between functional and nonfunctional tail genes cloned on high expression vectors. Phage tail gene mutations, generated in the cloned tail genes within these high expression vectors, resulted in the loss of the zone of lysis and inevitably DNA sequences determined from these "no zone" DNA plasmid mutants were found to contain mutations within the tail gene. To avoid titration of phage heads for each of the mutants isolated in the high expression vectors in the Berget Assay, these high expression vectors were transformed into strains other than BL21/DE3 to determine if there is enough residual tail gene expression to make them suitable for this modified Berget Assay. This residual expression in the absence of the host T7 RNA polymerase gene in the DE3 lambdoid phage is a well-known phenomenon among high expression vectors of the pET class but very few assays are sensitive enough to measure it. NovaBlue Mega (DE3/pET30aLIC-P22 TSP) transformants yielded zones of lysis. Site-directed mutagenesis and general plasmid mutagenesis [16,17] of these high expression plasmids generated "no zone mutants" with concomitant DNA changes in the tail gene. False positives have not yet been encountered. This assay's sensitivity may allow it to serve as a measure of "leakiness" for high expression vectors.

\subsection{Preparation Of Phage Heads (Tailless Phage)}

The preparation of phage heads was done as previously described [20].

\subsection{Mutagenic PCR Primers for Tail Gene Alterations}

The mutagenic primers, procured from IDT and used to create the pET11a-TSP mutant derivatives, are shown in Table 1. They have resulted in the generation of TSP with the following changes at the N-terminal domain: TSPALA6ASP, TSP-VAL9ASP and deletions of codons TSP$\Delta 2-15$, and TSP- $\Delta 2-23$. The transfers from primers to tail genes have been confirmed by DNA sequence analysis (Heflin DNA Sequencing Center at the University of Alabama).

\subsection{Mutagenic PCR Reactions}

Mutagenesis protocol was performed as described in the Stratagene QuikChange Mutagenesis Kit. In short, the plasmid, pET11a-TSP, which contains the P22 tail gene cloned into a high expression vector, is annealed to a mutagenic primer of between $30-36$ bases long and its reverse complement primer.

Table 1. Mutagenic primers used in this study

\begin{tabular}{|c|c|}
\hline Primer name & Primer DNA sequence* \\
\hline 5-A6D & ATG ACA GAC ATC ACT GAT AAC GTA GTT GTT TCT AAC \\
\hline 3-A6D & GTT AGA AAC AAC TAC GTT ATC AGT GAT GTC TGT CAT \\
\hline 5-VAL9ASP & ATC ACT GCA AAC GTA GAT GTT TCT AAC CCT CGT \\
\hline 3-VAL9ASP & ACG AGG GTT AGA AAC ATC TAC GTT TGC AGT GAT \\
\hline 5- del 2-15 & ATC TGG AGC AAA TTA AAT GAT CTT CAC TGA ATC C \\
\hline 3- del 15-2 & GGA TTC AGT GAA GAT CAT TTA ATT TGC TCC AGA T \\
\hline 5- del 2-23 & ATC TGG AGC AAA TTA AAT GAA AGC TGT TGC TAA T \\
\hline 3- del 23-2 & ATT AGC AAC AGC TTT CAT TTA ATT TGC TCC AGA T \\
\hline
\end{tabular}

*The mutagenic substitution is shown bolded and italicized for the coding 5' primer.

\subsection{Gene Expression Protocol}

Expression was carried out as described with BL21/DE3 (pET11-TSP) and its plasmid derivatives $[21,22]$. Cells were diluted and grown to an $\mathrm{OD}_{600}$ of $0.6-$
0.7 at $37^{\circ} \mathrm{C}$, whereupon, induction was initiated by the addition of IPTG to a final concentration of $1 \mathrm{mM}$ IPTG. Induction was allowed to continue for three hours at $30^{\circ} \mathrm{C}$. Aliquots of $1 \mathrm{~mL}$ were withdrawn from the uninduced culture (before the IPTG induction) and from the inducted 
cultures on the hour for the first three hours post-induction. At this time the cells were centrifuged and frozen as pellets at $-20^{\circ} \mathrm{C}$ till further use.

\subsection{SDS PAGE Analyses}

SDS PAGE analysis was done as described [23] using a BioRad mini vertical gel electrophoresis system. The discontinuous system of Laemmli was used with a $7.5 \%$ acrylamide separating gel. The electrophoresis run conditions were $100 \mathrm{~V}$ for 75 min by which time the BPB dye had reached the gel bottom and the run was terminated. Staining and destaining buffers have been described [24].

\section{Results and Discussion}

\subsection{Mutational Analyses}

To determine the possibility of a hydrophobic interaction between the stems and adjacent walls, the structure was screened for these pairs. It can be seen in Table 2 that each hydrophobic stem amino acid in column 1 can possibly interact with at least two hydrophobic amino acids located in the adjacent wall. The hypothesis is advanced that the three valine hydrophobic amino acids VAL8, VAL9 and VAL10 are critical in stabilizing the P22 TSP NTD by maintaining strong inter-chain hydrophobic contact with the adjacent chain. The middle codon was changed to aspartic acid. Since the patch may be the strongest interaction site with the adjacent chain, the other hydrophobic residue, ALA6 was not anticipated to account for much of that stability and its codon was also separately changed to ASP. These changes in the tail gene were anticipated to disrupt any putative hydrophobic interaction. In addition to these changes, the codons corresponding to the entire 23 amino acid stem (TSP $\Delta 2-23)$ and to the lower stem (TSP $\Delta 2-15)$ were deleted and transfer of all mutations to the pET11a-TSP plasmid DNA was confirmed by DNA sequence analysis Table 1 . Initial functional analysis was performed as detailed in the next section.

Table 2. Putative interactions between adjacent stem and wall amino acids

\begin{tabular}{|c|c|c|}
\hline $\begin{array}{c}\text { Amino acid in stem } \\
\text { (Chain D) }\end{array}$ & $\begin{array}{c}\text { Amino acid in } \\
\text { adjacent wall } \\
\text { (Chain F) }\end{array}$ & $\begin{array}{c}\text { Distance between } \\
\text { stem and wall* }\end{array}$ \\
\hline ALA6 & VAL83 & $5.67 \AA$ \\
\hline & THR84 & $4.79 \AA$ \\
\hline & VAL85 & $3.89 \AA$ \\
\hline & GLN86 & $7.00 \AA$ \\
\hline VAL8 & VAL83 & $5.50 \AA$ \\
\hline & THR84 & $5.26 \AA$ \\
\hline & VAL85 & $6.06 \AA$ \\
\hline VAL9 & GLN86 & $5.83 \AA$ \\
\hline & ILE82 & $5.96 \AA$ \\
\hline & VAL83 & $4.02 \AA$ \\
\hline VAL10 & THR84 & $6.15 \AA$ \\
\hline & & \\
\hline & ILE82 & $5.87 \AA$ \\
\hline
\end{tabular}

The closest adjacent hydrophobic amino acids chosen for study are in red color.

*The distances between amino acids were obtained from the Rasmol molecular visualization program from their "pick distance" function

\subsection{In vivo Assembly Using Stem Mutants (Berget Assay)}

The Berget Assay (in vivo functionality test) is used routinely to detect tail gene mutations. This assay is so accurate in determining functionality of a cloned tail gene that it is used as a screening tool to find mutants and suppressors. Representative results can be seen in Figure 3. Although this figure represents a screening assay where transformed candidates of a site-directed mutagenesis study were patched to determine if any had a zone of lysis or not (i.e. if the variant TSP was functional or not), the bottom of the plate contains information directly relevant to this study. Here the relevant data is found beneath and at either end of the longer horizontal line. On the left, it can be seen that the cloned gene for TSP-ALA6ASP gave a positive Berget Assay result, as was expected. The same TSP variant will at other times produce a third of the lysis area of the wild type clone. The Berget Assay is sensitive enough to be able to distinguish a partially defective TSP from WT TSP. On the right side of this figure the datum indicates that TSP-VAL9ASP was completely devoid of function and on the bottom below the small horizontal line is the control which is simply the pET11a vector alone. These data indicate that a single amino acid substitution in domain with subsequent proteolysis of the entire domain both deletions remove the essential VAL9, these deletion data are not surprising. These data do not tell us why the function is impaired. Nor is it known if the upper stem (aa15-aa23) is critical for function. Homology of three TSP NTDs from three different phages $\left(\varepsilon^{34}, \mathrm{P} 22\right.$ and Sf6 unpublished data) suggests that the two phenylalanines (PHE17 and PHE23) and amino acids 20-23 of the upper stem may be important since they are conserved in these three phages. These data indicate that part of this stem is critical for function. The data obtained indicate that the VAL9ASP mutation causes a critical flaw in TSP function while the ALA6ASP does not. At this qualitative level, the deletions of the lower stem and the upper stem are critical for function as indicated by their lack of function in the Berget Assay. Preliminary studies indicate that the other two amino acids in the valine patch, VAL8ASP and VAL10ASP, are devoid of tail function by the Berget Assay as well (J Williams and R Villafane, unpublished data).

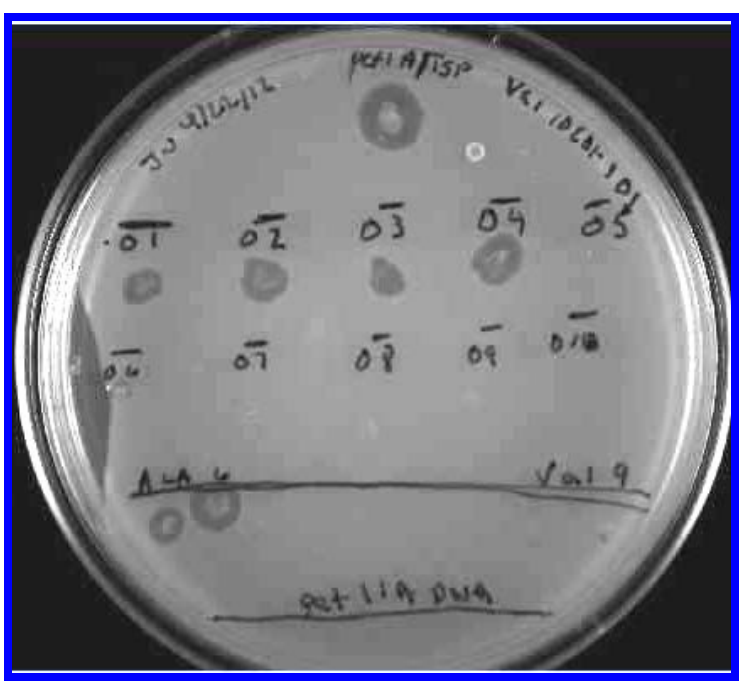

Figure 3. Berget Assay for screening of tailspike functionality. Ten candidates, labeled 01 to 10 , were screened for the lack of activity indicating the presence of mutations (Materials and Methods) 


\subsection{Protein Production of Stem Mutants Upon Induction of Gene Expression}

The Berget Assay indicated that all TSP variants, except ALA6ASP, could not support in vivo assembly (that is, the Berget Assay yielded a negative result: no area of lysis). To determine if the Berget negative results were due to proteolysis a gene expression study was initiated. Proteolysis may be indicated by a lack of protein induction. WT TSP, TSP-Val9Asp, TSP- $\Delta 2-15$ and TSP$\Delta 2$-23 were induced Figure 4 . Upon IPTG addition, the tail genes from WT and TSP- $\Delta 2-15$ were expressed and induced (Figure 4, lanes 2-3, 6-7; red arrow points to next lane which contains the induced protein). Figure 4 also shows that TSP-VAL9ASP and TSP- $\Delta 2-23$ mutant proteins were not induced or possibly degraded (Figure 4 , lanes 4-5, 8-9). A lack of induction may be due to a protein not being expressed, induced or being degraded. It is likely to have been expressed since this mutation was transferred by plasmid mutagenesis into the same vector as all the other variants and there is no evidence of differential transcriptional or translational control over the pET11a promoter for the TSP-VAL9ASP over the other constructed mutants. The pET11a-TSP plasmid has been extensive used with no report of differential protein expression. It is likely that the lack of induction was due to protein degradation. Comparison of the induction studies of TSP-VAL9ASP with TSP- $\Delta 2-15$ deletion is instructive (Figure 4, lanes 4-5, 6-7). The substitution mutant TSP-VAL9ASP did not induce tail protein expression whereas a truncated protein TSP- $\Delta 2-15$, whose deletion interval covers the same mutation interval, could be induced. The current interpretation of this result is that the VAL9 amino acid interaction is a very important or the major component in the stabilization interaction involving the hydrophobic patch with the adjacent chain. Its substitution by TSP-VAL9ASP removed this stabilizing interaction concomitantly grossly destabilizing the entire domain with subsequent proteolysis of the entire domain and possibly the entire TSP structure. However, removal of a fourteen amino acid segment including VAL9 restored some structural stability allowing for the induction of this protein. A possible explanation may be that the lower stem contains two amino acids types or interactions of opposing stabilizing functions. One amino acid type is involved in stabilizing this hydrophobic patch, of which VAL9 may be a member, and its substitution may lead to gross NTD destabilization while the other type inherently and naturally destabilizes this interaction. The mutation of the latter type should lead to stabilizing interactions. When the VAL9ASP is the only amino acid substitution present, it augments the destabilization already present by the second type of amino acids causing structural instability. The truncated protein removes both interactions types which may result in a stabilization of that protein structure and its ability to be induced. It should be pointed out that it is expected that in a low dielectric medium, as in the interior of the protein, where the stem is located, that the destruction of a hydrophobic interaction by introduction of a charged unpaired single charged residue would be thermodynamically unfavorable. Since proteins are only marginally stable, even interactions from individual amino acids can be critical [31,32,33]; Table 3.
Alternatively, it is also possible, but less likely because of the valine patch, that VAL9 may be important in the folding of this domain and its substitution alters this pathway and destabilizes this protein mutant. However, data concerning the folding of the P22 TSP NTD are lacking. The protein truncation of the entire stem structure, as represented by the TSP- $\Delta 2-23$ mutant protein, results in the lack of induction and/or proteolysis of the TSP. This result when considered with the fact that the TSP- $\Delta 2-15$ deletion does produce an induced protein, suggests that the upper stem does provide some stabilization. The deletion of the entire stem and its subsequent destabilization suggest that it is a major structural element involved in the stabilization of the trimer. The sum of the data suggests that the lower stem contributes the major stability. The data indicate that VAL9 is critical for stability but that both stem sections contribute to it.

\subsection{In vitro Assembly of Stem Mutants}

A more quantitative form of monitoring the interaction between the variant TSPs and the phage heads is the in vitro tailing or the assembly assay. To determine if the VAL9ASP (which produced no zone of lysis around the $E$. coli colony carrying the pET11a-TSP VAL9ASP) contained any residual head binding activity, the ability of it to assemble in vitro was tested. Table 3 (fourth column from left) indicates that there was no detectable assembly using the TSP-VAL9ASP extracts or separately for the TSP extract of the deletions as a source for TSP and as a consequence it has lowered the in vitro assembly seven orders of magnitude relative to the wild type TSP assembly reaction. This extremely large decrease in assembly can be due to the inability of the TSPVAL9ASP to bind to the phage heads as when the TSP NTD structure is altered or destroyed or if it does bind to the phage head, the production of a non-functional TSP. A variation of this assembly test was used to determine if the defective TSP-VAL9ASP and the deletion TSP variants bind to the phage heads in an unproductive manner. It has been termed "rescue of defective assembly pathway. The data for this rescue can be seen in Table 3 under the column "rescue". Addition of phage heads to extracts containing WT TSP reproduces the last step in phage assembly in vivo and produces infectious virus particles. If the defective TSP-VAL9ASP can bind to the phage heads, a reaction which is strong and irreversible, then subsequent addition of wild type TSP to the TSPVAL9ASP extract should cause no change in the phage titer as a result of assembly because the assembly TSP sites in the viral particle crevice will have been occupied by the mutant TSP. The converse is true if phage are produced which suggests that the NTD structure is somehow altered leaving its sites on the phage head unoccupied. Addition of WT TSP to the mutant TSPVAL9ASP extracts and to extracts containing TSP deletion variants leads to a significant increase in phage (Table 3, column 5). The study, done under conditions of phage head excess, produced phage particle suggesting that the TSP-VAL9ASP and the deletion TSP variants cannot bind to their cognate sites on the crevice formed by GP4 and GP10. This strongly suggests that the TSPVAL9ASP and deletion variant proteins are altered or degraded and unable to bind to the phage heads. This is 
also supported by the data from the assembly reaction Figure 4, Table 3.
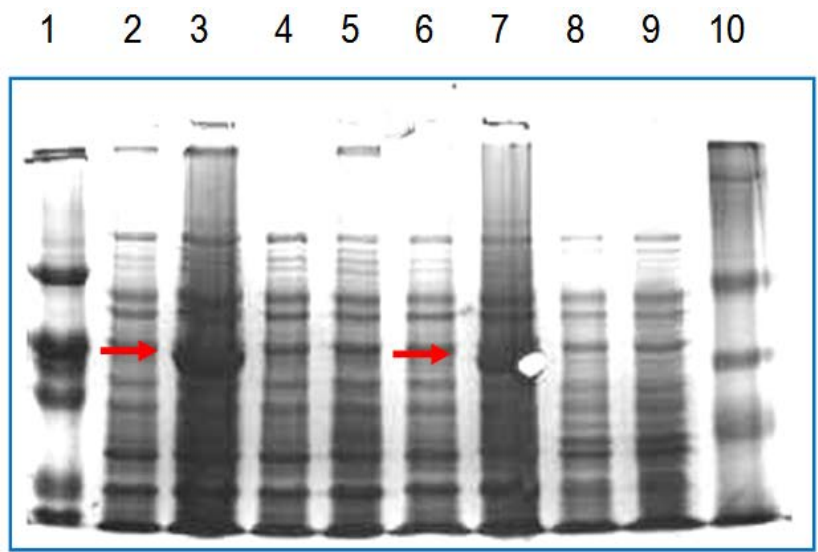

Figure 4. Protein Expression of BL21 (DE3/ pET11a-TSP and mutant derivatives, before and after IPTG induction. The red arrow indicates the position of the induced P22 TSP

Table 3. Comparison of TSP activities among WT and stem TSP variants

\begin{tabular}{|c|c|c|c|c|}
\hline $\begin{array}{l}\text { Stem amino } \\
\text { acid }\end{array}$ & $\begin{array}{l}\text { Berget } \\
\text { Assay }\end{array}$ & $\begin{array}{l}\text { Protein } \\
\text { induction }\end{array}$ & $\begin{array}{c}\text { In vitro } \\
\text { assembly } \\
\text { (pfu/mL) }\end{array}$ & $\begin{array}{l}\text { Assembly } \\
\text { rescue } \\
\text { (pfu/mL) }\end{array}$ \\
\hline WT & + & + & $5.4 \times 10^{7}$ & $\mathrm{n} / \mathrm{t}^{*}$ \\
\hline ALA6ASP & + & + & $\mathrm{n} / \mathrm{t}^{*}$ & $\mathrm{n} / \mathrm{t}^{*}$ \\
\hline VAL9ASP & -- & -- & 0 & $7.0 \times 10^{6}$ \\
\hline$\Delta 2-15$ & -- & + & $1.3 \times 10^{3}$ & $1.6 \times 10^{8}$ \\
\hline$\Delta 2-23$ & -- & -- & 0 & $1.2 \times 10^{8}$ \\
\hline
\end{tabular}

This report suggests that a short stretch of amino acids located within the first fifteen amino acids of the P22 TSP NTD is critical for the stability of the dome structure formed by the first 108aa of the P22 TSP NTD. The first 23aa are located within this dome-like structure and have been dubbed the "stem" of the NTD. Although the first 15aa (lower stem) are critical, especially Val9, deletion analysis and in vitro assembly studies implicate the rest of the stem in additional stabilizing interactions. This study indicates that this P22 TSP NTD is a suitable model for the study of protein-protein interactions and protein stability as they are one and the same in this particular study.

\section{Acknowledgement}

The authors thank Dr Peter Prevelige (Dept of Microbiology, University of Alabama at Birmingham) for the BL21 (DE3/pET11aTSP) which plasmid, pET11aTSP, contains the cloned wild type version of the P22 tail gene, from which all mutant derivatives are being derived. We thank Dr Jon King and Cameron Haase-Pettingell (Biology Department, MIT) for bacterial strains and for the lysogenic strain containing the P22 phage mutant from which we prepared phage heads which were used in our Berget and in vitro assembly assays, DB7136 (9-amN110/ 13-amH101/ c2ts30). Finally these studies were supported by the Deanship of the College of Science, Mathematics and Technology at the Alabama State University and by the Department of Chemistry and Biochemistry at Huntingdon College.

\section{References}

[1] Campbell, A., "Comparative molecular biology of lambdoid phages," Annual Review of Microbiology, 48,193-222, 1994.

[2] Casjens, S.R., Thuman-Commike, P.A., "Evolution of mosaically related tailed bacteriophage genomes seen through the lens of phage P 22 virion assembly,” Virology, 411, 393-415, 2012.

[3] Botstein, D., Waddell, C. H., King J., "Mechanism of head assembly and DNA encapsulation in Salmonella phage P 22. I. Genes, proteins, structures and DNA metabolism," Journal of Molecular Biology, 80, 669-695, 1973.

[4] King, J., Lenk, E. V., Botstein, D., "Mechanism of head assembly and DNA encapsulation in Salmonella phage P 22. II. Morphogenetic pathway," Journal of Molecular Biology, 80, 697731, 1973.

[5] Hartwieg, E., Bazinet, C., King, J., "DNA injection apparatus of phage P22," Biophysical Journal, 49, 24-26, 1984.

[6] Chiang, J., Weigele, P., King, J., Chiu, W., Jiang, W., "Cryo-EM asymmetric reconstruction of bacteriophage $\mathrm{P} 22$ reveals organization of its DNA packaging and infecting machinery," Structure, 14, 1073-1082, 2006.

[7] Lander, G. C., Khayat, R., L,i R., Prevelige, P.E., Potter, C. S., Carragher, B., Johnson, J. E., "The P 22 tail machine at subnanometer resolution reveals the architecture of an infection conduit," Structure, 17, 789-798, 2009.

[8] Lander, G.C., Tang, L., Casjens, S.R., Gilcrease, E. B., Prevelige, P., Poliakov, A., Potter, C. S., Carraher, B., Johnson, J. E., "The structure of an infectious P 22 virion shows the signal for headful DNA packaging," Science, 312, 1791-1795, 2006.

[9] Steinbacher, S., Miller, S., Baxa, U., Budisa, N., Weintraub, A., Seckler, R., Huber, R., "Phage P 22 tailspike protein: Crystal structure of the head-binding domain at $2.3 \AA$, fully refined structure of the endorhamnosidase at $1.56 \AA$ resolution, and the molecular basis of O-antigen recognition and cleavage,” Journal of Molecular Biology, 267, 865-880, 1997.

[10] Steinbacher, S., Seckler, R., Miller, S., Streipe, B., Huber, R., Reinemer, P., "Crystal structure of the P 22 tailspike protein: Interdigitated subunits in a thermostable trimmers," Science, 265, 383-386, 1994.

[11] The PyMOL Molecular Graphics System, Version 1.5.0.4 Schrödinger, LLC.

[12] Israel, J. V., Anderson, T. F, Levine, M., "In vitro morphogenesis of phage P 22 from phage heads and base-plate parts," Proceedings of the National Academy of Sciences, 57, 284-291, 1967.

[13] Schwarz, J. J., Berget, P. B., "The isolation and sequence of missense and nonsense mutations in the cloned bacteriophage P 22 TSP gene," Genetics, 121, 635-649, 1989.

[14] Villafane, R., Costa, S., Ahmed, R., Salgado, C., "Conservation of the N-terminus of some phage tails proteins," Archives of Virology, 150, 2609-2621, 2005.

[15] Robinson, A. S., King, J., "Disulfide-bonded intermediate on the folding and assembly pathway of a non-disulfide bonded protein," Nature Structural Biology, 4, 450-455, 1997.

[16] Sambrook, J., Fritsch, E. F., Maniatis, T., Molecular Cloning: A Laboratory Manual, $2^{\text {nd }}$ edition, CSH Press, New York, 1989.

[17] Villafane, R., "Construction of phage mutants," Methods in Molecular Biology, 501, 223-237, 2009.

[18] Villafane, R., Fleming, A., Haase-Pettingell, C., "Isolation of suppressors of temperature-sensitive folding mutants," Journal of Bacteriology, 176:137-142, 1994.

[19] Berget, P. B., Poteete, A. R., Sauer, R. T., "Control of phage P 22 tail protein expression by transcription termination," Journal of Molecular Biology, 164, 561-572, 1983.

[20] Kamei, D.T., Liu, C., Haase-Pettingell, C., King, J. A., Wang, D. I. C., Blankschtein, D., "Understanding viral partitioning in twophase nonionic micellar systems: Role of attractive interactions between viruses and micelles," Biotechnology and Bioengineering, 78, 190-202, 2002.

[21] Haase-Pettingell, C., Betts, S., Raso, S.W., Stuart, L., Robinson, A., King, J., "Role of cysteine residues in the in vivo folding and assembly of the phage P 22 tailspike,” Protein Science, 10, 397410, 2001.

[22] Weigele, P. R., Haase-Pettingell, C., Campbell, P. G., Gossard, D.C., King, J. "Stalled folding mutants in the triple beta -helix domain of the phage P 22 tailspike adhesion," Journal of Molecular Biology, 354, 1103-1117, 2005. 
[23] Copeland, R.A. Methods for Protein Analysis: A Practical Guide To Laboratory Protocols, Chapman and Hall Press, New York, 1994, pp. 59-98.

[24] Roskams, J., Rodgers, L., Electrophoretic separation of proteins and nucleic acids. In: Roskams J, Rodgers L (eds), Lab Ref: A handbook of recipes, reagents, and other reference tools for use at the bench. Cold Spring Harbor Press, New York, 2002, pp. 63-91.

[25] Chandler, D., "Interfaces and the driving force for hydrophobic assembly,” Nature, 437, 640-647, 2005.

[26] Pace, C. N., Fu, H., Fryar, K. L., Landua, J., Trevino, S.R., Shirley, B.A., Hendricks, M.M., Iimura, S., Gajiwa, K., Scholtz, J. M., Grimsley, G. R., "Contribution of hydrophobic interaction to protein stability," Journal of Molecular Biology, 408, 514-528, 2011.

[27] Saha, R. P., Bahardur, R. P., Chakrabarti, P., "Interresidue contacts in proteins and protein-protein interfaces and their use in characterizing the homodimeric interface," Journal of Proteome Research, 4, 1600-1609, 2005.
[28] Tsai, C-J., Lin, S. L., Wolfson, H.J., Nussinov, R., "Studies on protein-protein interfaces: A statistical analysis of the hydrophobic effect," Protein Science, 6, 53-64, 1997.

[29] Moreira, I. S., Fernandes, P.A., Ramos, M. J., "Hot spots-A review of the protein-protein interface determinant amino acid residues," Proteins, 68, 803-812, 2007.

[30] Glaser, F., Steinberg, D.M., Vakser, I. A., Ben-Tal, N., "Residue frequencies and pairing preferences at protein-protein interfaces," Proteins, 43, 89-102, 2001.

[31] Keskin, O., Gursoy, A., Ma, B., Nussinov, R., "Principles of protein-protein interactions: What are the preferred ways for proteins to interact?" Chemical Reviews, 108, 1225-1244, 2008.

[32] Dill, K. A., "Dominant forces in protein folding," Biochemistry, 29, 7133-7155, 1990.

[33] Maurides, P.A., Schwarz, J. J., Berget, P. B., "Intragenic suppression of a capsid-assembly defective P 22 tailspike mutation”, Genetics, 125, 673-681, 1989. 